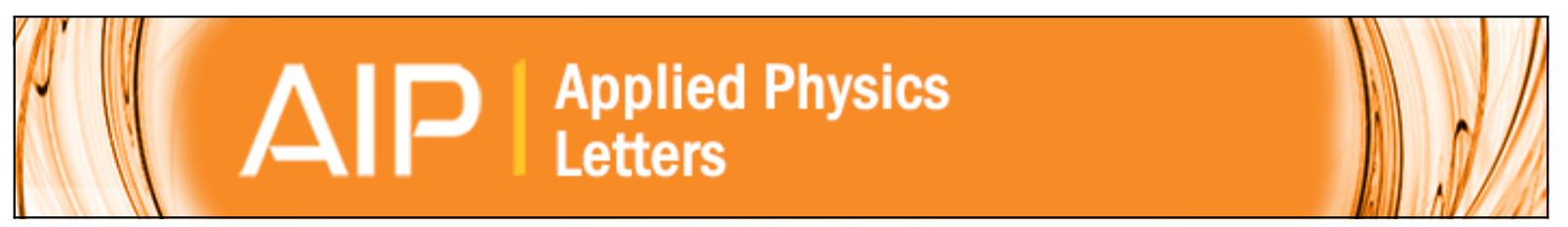

\title{
Electron-beam driven relaxation oscillations in ferroelectric nanodisks
}

Nathaniel Ng, Rajeev Ahluwalia, Ashok Kumar, David J. Srolovitz, Premala Chandra, and James F. Scott

Citation: Applied Physics Letters 107, 152902 (2015); doi: 10.1063/1.4932653

View online: http://dx.doi.org/10.1063/1.4932653

View Table of Contents: http://scitation.aip.org/content/aip/journal/apl/107/15?ver=pdfcov

Published by the AIP Publishing

\section{Articles you may be interested in}

Characterization of electron-beam recorded microdomain patterns on the nonpolar surface of LiNbO3 crystal by nondestructive methods

Appl. Phys. Lett. 105, 142901 (2014); 10.1063/1.4897279

Nanoscale domain patterning of lead zirconate titanate materials using electron beams

Appl. Phys. Lett. 84, 774 (2004); 10.1063/1.1644327

Lead oxide nanobelts and phase transformation induced by electron beam irradiation

Appl. Phys. Lett. 80, 309 (2002); 10.1063/1.1432749

Size effects on ferroelectricity of ultrafine particles of $\mathrm{PbTiO} 3$

J. Appl. Phys. 87, 3462 (2000); 10.1063/1.372367

The role of lead component in second-harmonic generation in lead silica by electron-beam irradiation

Appl. Phys. Lett. 73, 3040 (1998); 10.1063/1.122665

\section{AIP $\left.\right|_{\text {APL Photonics }}$}

APL Photonics is pleased to announce Benjamin Eggleton as its Editor-in-Chief

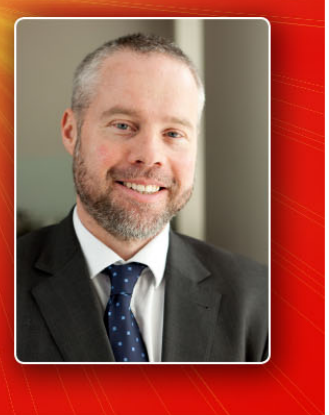




\title{
Electron-beam driven relaxation oscillations in ferroelectric nanodisks
}

\author{
Nathaniel Ng, ${ }^{1}$ Rajeev Ahluwalia, ${ }^{1}$ Ashok Kumar, ${ }^{2}$ David J. Srolovitz, ${ }^{3}$ Premala Chandra, ${ }^{4}$ \\ and James F. Scott ${ }^{5,6}$ \\ ${ }^{1}$ Institute of High Performance Computing, Singapore 138632, Singapore \\ ${ }^{2}$ CSIR-National Physical Laboratory, Delhi 110012, India \\ ${ }^{3}$ Department of Materials Science and Engineering and Department of Mechanical Engineering \\ and Applied Mechanics, University of Pennsylvania, Philadelphia, Pennsylvania 19104, USA \\ ${ }^{4}$ Department of Physics and Astronomy, Center for Materials Theory, Rutgers University, \\ Piscataway, New Jersey 08854, USA \\ ${ }^{5}$ Department of Physics, Cavendish Laboratory, J. J. Thompson Avenue, Cambridge CB3 OHE, \\ United Kingdom \\ ${ }^{6}$ Department of Chemistry and Department of Physics, University of St. Andrews, St. Andrews YX16 9ST, \\ United Kingdom
}

(Received 21 July 2015; accepted 27 September 2015; published online 15 October 2015)

\begin{abstract}
Using a combination of computational simulations, atomic-scale resolution imaging and phenomenological modelling, we examine the underlying mechanism for nanodomain restructuring in lead zirconate titanate nanodisks driven by electron beams. The observed subhertz nanodomain dynamics are identified with relaxation oscillations where the charging/discharging cycle time is determined by saturation of charge traps and nanodomain wall creep. These results are unusual in that they indicate very slow athermal dynamics in nanoscale systems, and possible applications of gated versions are discussed. (C) 2015 AIP Publishing LLC. [http://dx.doi.org/10.1063/1.4932653]
\end{abstract}

The importance of surfaces and sample size in determining static polarization configurations has been emphasized in flux-closure ${ }^{1-5}$ and faceted ${ }^{6-8}$ domain pattern studies in several ferroelectric materials. Recently, it has become possible to reorient domains and defects on nanoscales with electron beams, e.g., employing high resolution transmission electron microscopy (HRTEM). ${ }^{9-11}$ Nanodomain dynamics in ferroelectric nanodisks can also be probed with real-time HRTEM, and have been observed to be considerably slower $(10 \mathrm{~s})^{11}$ than those in their nanomagnetic counterparts $(50 \mathrm{~ns}) .{ }^{12}$ Although in principle such slow nanodomain reorientation could arise from thermal effects, it has been shown that such heating is negligible under experimental conditions such as employed in the present study. ${ }^{13}$ In this paper, we identify a physical mechanism based on relaxation oscillations with a charging/discharging cycle to explain the ten orders of magnitude separating the nanodynamics of ferroelectric nanodisks and their ferromagnetic counterparts. Such slow cycling times are highly unusual in nanoscale objects, and possible applications of gated versions are discussed.

Here, we show that driven domain dynamics in freestanding ferroelectric nanostructures are strongly influenced by electrostatic conditions. In a combined computational, experimental, and phenomenological effort, we identify the charging/discharging mechanism underlying the observed oscillations between circular and hexagonal nanodomain patterns in lead zirconate titanate (PZT) nanodisks driven by electron beams. Using phase-field modelling, ${ }^{10,14,15}$ we demonstrate the crucial influence of charge on the nanodomain patterns, and show that we can reproduce domain structures similar to those observed in experiment. The subhertz frequency response of the nanodomain reorientation results from relaxation oscillations ${ }^{16-18}$ between threshold charging of surface traps ${ }^{19}$ and subsequent discharging by nanodomain wall creep, ${ }^{20}$ where quantitative agreement is made using prior measurements on PZT samples.

Experimentally, edge-supported 8-nm diameter disks of PZT were exposed to electron beams with a $0.5 \mathrm{~A} / \mathrm{m}^{2}$ probe current density; further details can be found elsewhere. ${ }^{11}$ The samples exhibit two distinct structures between which the sample "flopped;" a nearly circular disk shape with nanodomain walls perpendicular to the disk perimeter and a faceted disk shape with the nano domain walls parallel to the disk perimeter, as displayed in Figures 1(a) and 1(c). Since the HRTEM only reveals ferroelastic (rather than ferroelectric) domain walls, modelling is needed to interpret the role played by charging the electron beam that drives the observed faceting oscillations and the simultaneous realignment of nanodomain walls. It is known that electron beams can lead to charging of insulating materials. ${ }^{21}$ It has been shown that electron beams can be used to switch the polarization direction in ferroelectric ${ }^{22}$ and can even stabilize unusual quadrant patterns in free standing nanodisks. ${ }^{10}$ In order to investigate e-beam induced charging effects in ferroelectric nanodisks, we use a phase-field approach to simulate how domain patterns are influenced by radial fields generated by uniform free charge. The model, based on earlier studies, ${ }^{10,15,23}$ has been adapted to incorporate the nano disk geometry (see supplementary material ${ }^{24}$ ) of the experiment; the goal is to determine whether the observed nanodomain pattern realignment is driven by depolarization fields in a charging/discharging cycle. We should point out that although in the original experiments a composition where PZT is a mixture of tetragonal phases was chosen, for simplicity, we restrict our model to the case where tetragonal is the only stable phase. Our aim is to model and understand the basic mechanism behind the faceting oscillation. We believe that the observed phenomenon is generic and does not depend on the underlying symmetry of the crystal. 

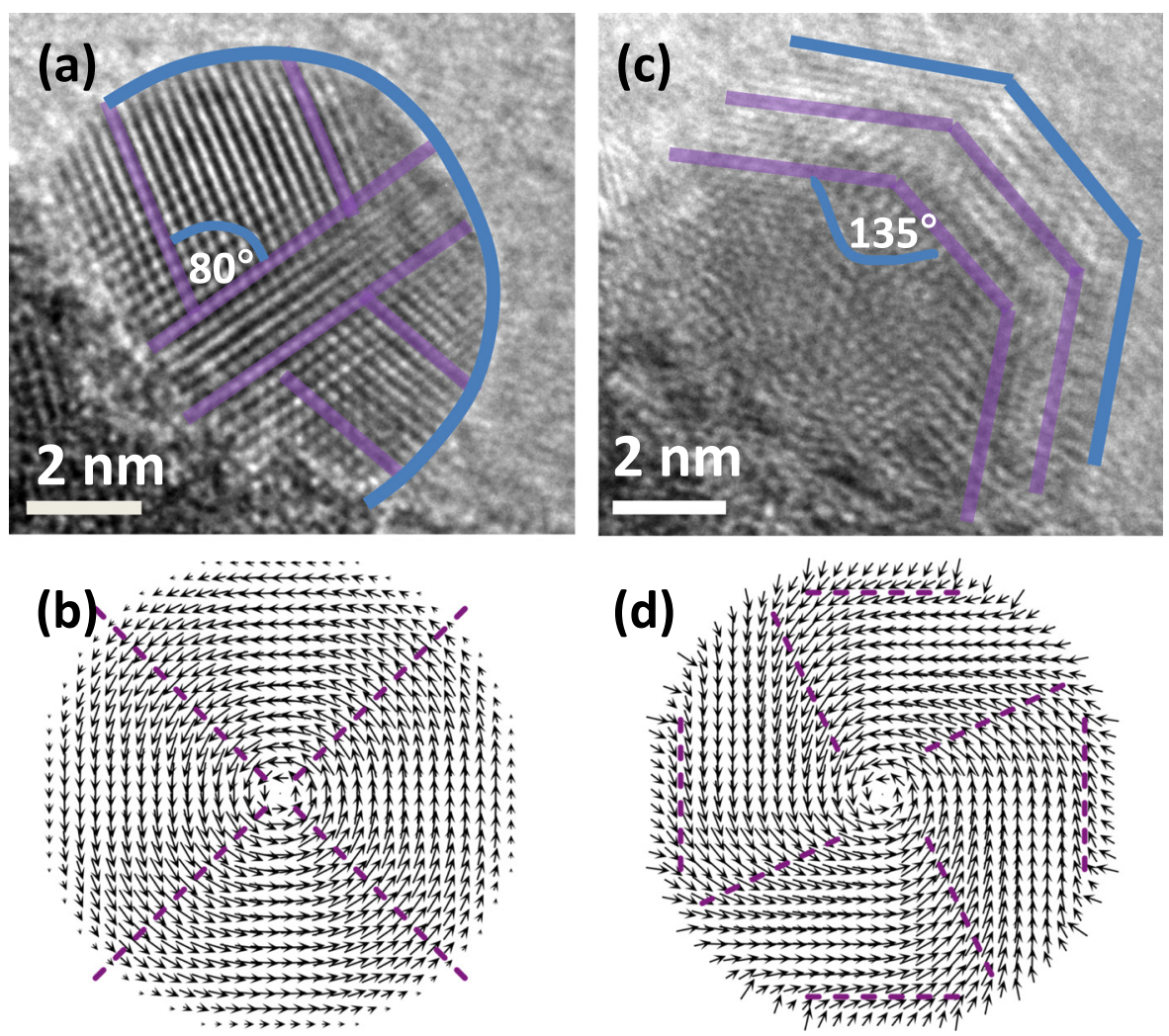

FIG. 1. HRTEM images for (a) circular (unfaceted) and (c) faceted 8-nm PZT nanodisks; the ferroelastic domain walls are illustrated as purple lines and the angles between them are indicated. Polarization vectors from simulations for a 16-nm nanodisk for (b) uncharged and (d) charged nanodisks. Dashed purple lines in (b) and (d) indicate the approximate positions of ferroelastic domain walls. Note that while domain walls are perpendicular to the perimeter in (b), additional ferroelastic domain walls parallel to the perimeter are observed in (d).
We first simulate the uncharged case by solving the phase field equations for a $16 \mathrm{~nm}$ nanodisk quenched from the paraelectric state. Note that in order to improve the resolution of our simulations, we have considered a diameter larger than that of the nanodisk in the experiments. We have confirmed that this increase in diameter does not change the conclusions of the paper. A uniform charge density, $N_{e}=4$ $\times 10^{26} \mathrm{~m}^{-3}$, is then applied and the evolution of the domains is monitored. Images are generated using Matplotlib. ${ }^{25}$ Fig. 1(b) shows the simulated pattern for the uncharged case with a flux closure domain pattern where the dashed purple lines indicate the positions of the ferroelastic domain walls, normal to the disk perimeter. This is consistent with the domain wall configuration in the HRTEM image in Fig. 1(a), suggesting that it corresponds to an uncharged disk.

Figure 1(d) shows the nanodomain pattern in the charged nanodisk. Similar to Fig. 1(c), the approximate positions of the ferroelastic domain walls are shown by dashed purple lines. For this case, the flux closure domain pattern transforms to one where polarization vectors have a non-zero radial component at the perimeter and a polarization vortex type state (characterized by a vanishing radial component in the center). This leads to the additional ferroelastic domain walls that are parallel to the disk perimeter. This behavior may be understood in terms of the radial electric field that vanishes at the center, which arises due to the uniformly charged density associated with the electron beam induced charging. ${ }^{10}$ Vortex formation at the center occurs to avoid head-to-head polarization, resulting in areas having $\mathbf{P} \cdot \mathbf{n}=0$ (center) and $\mathbf{P} \cdot \mathbf{n} \neq 0$ along the perimeter, where the radial unit vector $\mathbf{n}=\frac{\mathbf{r}-\mathbf{r}_{0}}{\left|\mathbf{r}-\mathbf{r}_{0}\right|}$ and $\mathbf{r}_{0}=\left(x_{0}, y_{0}\right)$ represents the coordinates of the centre of the disc. Regions of distinct $\mathbf{P} \cdot \mathbf{n}$ are separated by ferroelastic nanodomain walls that can be observed in the HRTEM. This is further illustrated in the distributions of $\mathbf{P} \cdot \mathbf{n}$ in Fig. 2. Note that due to the underlying crystalline anisotropy, the $\mathbf{P} \cdot \mathbf{n}$ distribution is strongly anisotropic.

The additional domain walls in Figs. 1(d) and 2 which are aligned along the disk perimeter are consistent with Fig. 1(c) where domain walls are observed parallel to the faceted edges. Thus, this situation corresponds to the HRTEM image in Fig. 1(c), indicating that it results from nanodisks with charged boundaries.

Our phase-field simulations indicate that this domain wall realignment occurs between charged and uncharged states of the PZT nanodisk. While this clarifies why electron beam induced charging leads to domain wall realignment, it does not directly account for the observed nanodisk faceting that accompanies it. To understand this, we first note that crystalline anisotropy leads to the formation of straight/flat domain walls. It is not possible to maintain both straight domain walls (as favored by crystalline anisotropy) and domain walls parallel to the nanodisk perimeter (as favored by the radial electric field associated with charging) when the nanodisk perimeter is curved. Faceting of the nanodisk provides a solution that allows for straight domain walls parallel to the nanodisk perimeter. This situation is schematically depicted in Fig. 2(b) where we show how ferroelastic domain walls parallel to the free surface can lead to the faceting of the disk into a polygon. While a full thermodynamic analysis of the faceting requires consideration of surface energy anisotropy, the effects of nanodisk shape (boundary conditions) on the electrostatic (ferroelectric) and elastic (ferroelastic) fields which are beyond the scope of this study, these considerations suggest that faceting is a consequence of the charginginduced realignment of the domain walls.

This electrostatic analysis accounts for the two observed nanodomain configurations, but suggests a frequency response on a gigahertz scale in contrast to the subhertz 


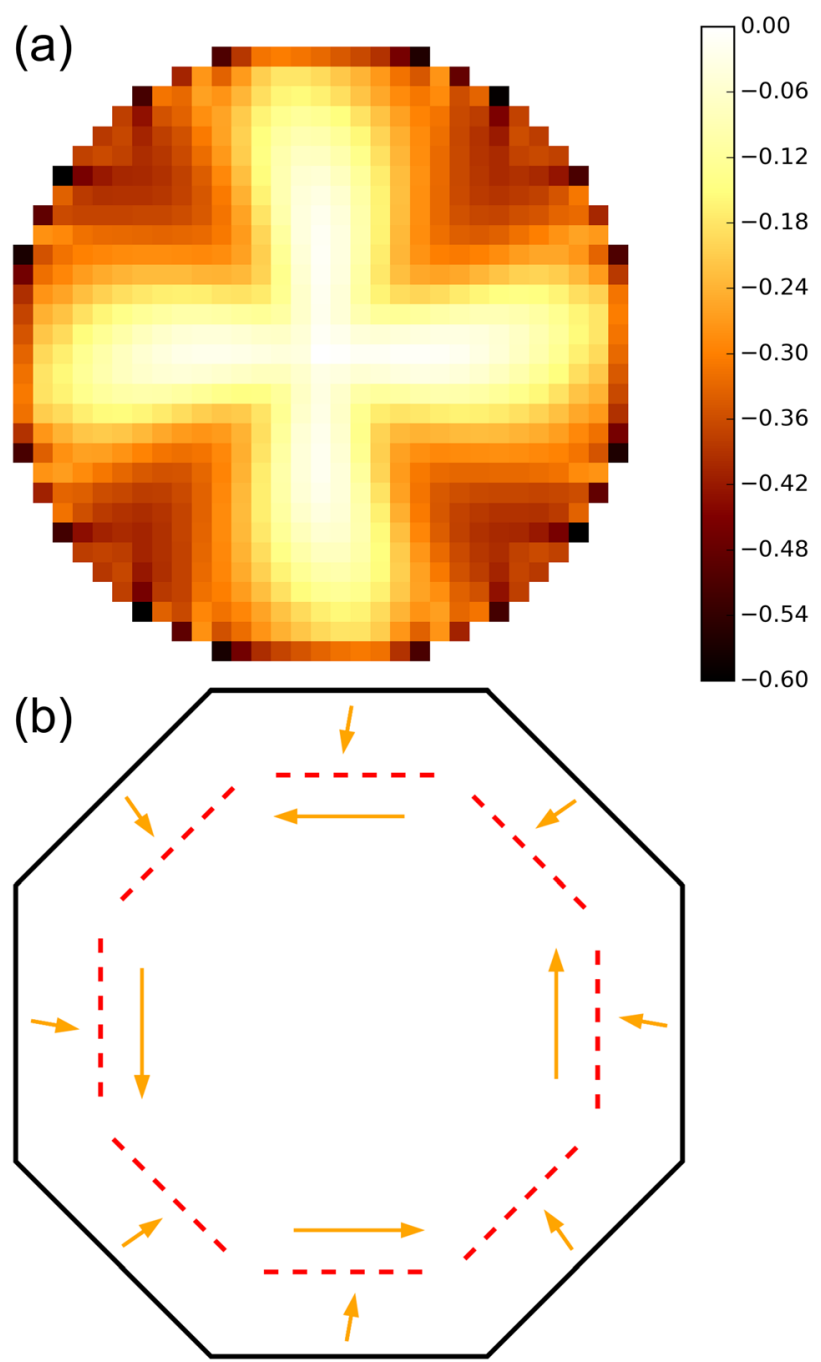

FIG. 2. (a) Distribution of the normalized radial component of the polarization, $\frac{1}{P_{s}} \mathbf{P} \cdot \mathbf{n}$ corresponding to Fig. 1(d). The radial component of the polarization is zero in the center and nonzero towards the edges. Note that the distribution shows strong anisotropy (i.e., $\theta$-dependence). (b) Schematic showing the ferroelastic domain walls (dotted red lines) and the associated faceted perimeter.

frequencies observed. ${ }^{11}$ Because thermal effects have been ruled out for these experimental conditions, ${ }^{13}$ this slow timescale suggests extreme nonlinearity and possibly mechanical overshoot. Our phase-field modelling indicates a driven and reversible charging/discharging cycle that is naturally described as an effective relaxation oscillation circuit. This is a system that is intrinsically out-of-equilibrium: it cycles between an unstable state and a frustrated attempt to reach equilibrium that is prevented by onset behavior of its nonlinear element that forces it back to its original unstable state. ${ }^{17,18}$ Electronically, this is akin to a nonlinear circuit element, such as a neon light or a unijunction transitor, with a threshold voltage for negative resistance in parallel with a conducting channel (usually a capacitor).

Self-sustaining subhertz oscillations in a physical system were reported in a wide-gap semiconductor $\mathrm{ZnO},{ }^{16}$ and we propose that the e-beam experiments on PZT nanodisks also present such an example. It is known that electron beam irradiation creates charge traps and defects in semiconducting targets. The time-scale for the charging cycle will then be set by the charge saturation of these surface states. This charge storage threshold in PZT targets exposed to electron beams has been measured quantitatively to be $5 \mathrm{C} / \mathrm{m}^{2}$. For the experiments of interest here, ${ }^{11}$ with a current density of $0.5 \mathrm{~A} / \mathrm{m}^{2}$, this corresponds to a charging time of

$$
\tau_{\text {charging }}=\frac{5 \mathrm{C} / \mathrm{m}^{2}}{0.5 \mathrm{C} / \mathrm{s}-\mathrm{m}^{2}}=10 \mathrm{~s}
$$

that is the same order of magnitude as the observed faceting oscillations. ${ }^{11}$ We note that if we assume that these traps are singly charged, then there are $\frac{5 \mathrm{C} / \mathrm{m}^{2}}{1.6 \times 10^{-19} \mathrm{C} / \mathrm{e}}=3 \times 10^{19} \mathrm{traps} / \mathrm{m}^{2}$ that is consistent with concentrations reported for oxygen vacancies in commercial quality spin-on PZT films; ${ }^{26}$ this value is also in reasonable agreement with the electron concentration used in our simulations $\left(N_{e}=4 \times 10^{26} \mathrm{~m}^{-3}\right)$.

Finally, we note that our system is a charge analogue of a seesaw with a faucet continuously dripping water into a container on one end, and a block on the other one. This can be analyzed as a mechanical relaxational oscillator ${ }^{27}$ in which the seesaw flips when the weight of the water container is greater than that of the block; however, once the container touches the ground it empties, the seesaw goes back to its original position, and the whole process starts again. In the context of the present work, the electron beam is analogous to the water flow from the faucet; the threshold is related to the Zener breakdown ${ }^{28}$ that occurs in pnjunctions at low fields ca. $E \ll 1 \frac{\mathrm{v}}{\mathrm{nm}}$ and involves excitation of electrons into the conduction band from the valence band or trap states within the bandgap. It is non-destructive and reversible, and thus differs from avalanche breakdown that occurs at higher fields.

The charging of the nanocrystal creates depolarization fields that realign the domain walls and leads to the observed faceting (Fig. 1). During the initial part of the cycle, the current from the electron beam is filling the surface states with charge and the nanodisk behaves like a capacitor. However, once the charge saturation threshold for these traps and defects in the PZT nanodisk is reached, ${ }^{19}$ the depolarization fields become strong enough to initiate the current flow in the nanodisk. This current leads to discharging and the subsequent decrease of the depolarization field; consequently, the domain walls realign back to their uncharged configurations and the nanodisk returns to its original circular shape. The presence of surface states and large electromechanical coupling means that this involves domain creep in a random elastic medium that has been discussed quantitatively and measured in the PZT films. ${ }^{20}$ For the experimental parameters of interest, the domain wall creep in the PZT epitaxial films is found to be $v=10^{-9} \mathrm{~m} / \mathrm{s}$ (Ref. 20) so that for the dots of roughly $10 \mathrm{~nm}$ (of thickness $100 \mathrm{~nm}$ ), the discharging time-scale is

$$
\tau_{\text {discharging }}=\frac{10 \mathrm{~nm}}{10^{-9} \mathrm{~m} / \mathrm{s}}=10 \mathrm{~s} .
$$

This is again of the same order of magnitude as the observed time-scale. This charging/discharging cycle is expected to continue as long as the electron beam is on and there is a continuous flow of charge into the nanodisk. In contrast with many relaxation oscillators, in these PZT nanodisks there are 
slow time-scales associated with both the charging and the discharging cycles where the rate-limiting one is clearly the slower of the two.

Thus, the cycling times, achieved both by macroscopic (charging threshold) and microscopic (discharging via domain creep) mechanisms, is consistent with the experimental observations. We note here that the two underlying phenomena, charge trapping and slow domain creep due to electromechanical coupling and mass, are both absent in magnetism which explains the many orders of magnitudes that separate the frequency response of field-driven ferroelectric and ferromagnetic nanodisks.

In summary, we have studied the mechanism underlying nanodomain restructuring in the PZT nanodisks driven by electron beams. Our phase-field modelling identifies the two observed nanodomain patterns associated with the uncharged (domain walls perpendicular to the perimeter) and charged (domain wall parallel to the perimeter) free-standing nano disks. Faceting in the changed sample is the result of the crystalline anisotropy requirement of straight domain walls and the tendency for the domain walls to align parallel to the surface.

The observed subhertz frequency response, many orders of magnitude lower than expected from an electronic mechanism, is a form of relaxation oscillations, where the charging/discharging times are determined by charge trap saturation and nanodomain wall creep. We predict that the oscillation time-scale can be tuned by changing the surface trap density, either by varying the beam current or by annealing the PZT nanodisks in oxygen/ozone environments. Though this charging/discharging cycle here is driven by electron beams, similar behavior could be achieved by gating the PZT nanodisks to achieve a voltage-controlled sourcegate-drain device. It would be unusual to have such small semiconductor triodes with such slow time-scales; these could be important for biological applications like medical implants where time-scales are naturally of the order of seconds. We also note that relaxation oscillators are common in commercial power inversion devices, so these triodes could also have application as nanoscale dc to ac converters.

This work was supported by the A*STAR Computational Resource Centre through the use of its high performance computing facilities and by National Science Foundation Grant No. NSF-DMR-1334428 (P. Chandra). P.C. is grateful for the hospitality of Trinity College,
Cambridge where part of this work was performed. We thank Robert Laskowski for useful discussions.

${ }^{1}$ I. Naumov, L. Bellaiche, and H. Fu, Nature 432, 737 (2004).

${ }^{2}$ P. Aguado-Pente and J. Junquera, Phys. Rev. Lett. 100, 177601 (2008).

${ }^{3}$ C.-L. Jia, K. Urban, M. Alexe, D. Hesse, and I. Vrejoiu, Science 331, 1420 (2011).

${ }^{4}$ L. McGilly and J. Gregg, Nano Lett. 11, 4490 (2011).

${ }^{5}$ Y. Tang, Y. Zhu, X. Ma, A. Borisevich, A. Morozovska, E. Eliseev, W. Wang, Y. Wang, Y. Xu, Z. Zhang, and S. Pennycock, Science 348, 547 (2015).

${ }^{6}$ C. Ganpule, A. Roytburd, V. Nagarajan, B. Hill, S. Ogale, E. Williams, R. Ramesh, and J. Scott, Phys. Rev. B 65, 014101 (2001).

${ }^{7}$ J. F. Scott, A. Gruverman, D. Wu, I. Vrejoiu, and M. Alexe, J. Phys.: Condens. Matter 20, 425222 (2008).

${ }^{8}$ I. Lukyanchuk, P. Sharma, T. Nakajima, S. Okamura, J. Scott, and A. Gruverman, Nano Lett. 14, 6931 (2014).

${ }^{9}$ A. W. Robertson, C. S. Allen, Y. A. Wu, K. He, J. Oliver, J. Neethling, A. I. Kirkland, and J. H. Warner, Nat. Commun. 3, 1144 (2012).

${ }^{10}$ R. Ahluwalia, N. Ng, A. Schilling, R. G. P. McQuaid, D. M. Evans, J. M. Gregg, D. J. Srolovitz, and J. F. Scott, Phys. Rev. Lett. 111, 165702 (2013).

${ }^{11}$ J. F. Scott and A. Kumar, Appl. Phys. Lett. 105, 052902 (2014).

${ }^{12}$ A. Bisig, M. Stärk, M.-A. Mawass, C. Moutafis, J. Rhensius, J. Heidler, F. Buttner, M. Noske, M. Weigand, S. Eisebitt, T. Tyliszczsk, B. Van Waeyenberge, H. Stoll, G. Schultz, and M. Klaui, Nat. Commun. 4, 2328 (2013).

${ }^{13}$ H. Zheng, Y. Liu, F. Cao, S. J. Wu, S. F. Jia, A. J. Cao, D. S. Zhao, and J. B. Wang, Sci. Rep. 3, 1920 (2013).

${ }^{14}$ S. Sriram, M. Bhaskaran, R. Ahluwalia, T. G. Nguyen, N. Ng, D. J. Srolovitz, K. Kalantar-zadeh, and A. Mitchell, ACS Nano 5, 1067-1072 (2011).

${ }^{15}$ N. Ng, R. Ahluwalia, and D. J. Srolovitz, Acta Mater. 60, 3632-3642 (2012).

${ }^{16}$ O. V. Losev, Radio News 6, 1287 (1925), see http://www.americanradiohistory. com/Archive-Radio-News/20s/Radio-News-1925-01-R.pdf.

${ }^{17}$ B. van der Pol, Philos. Mag. 2, 978 (1926).

${ }^{18}$ A. Pippard, The Physics of Vibrations (Cambridge University Press, 1979).

${ }^{19}$ D. Li, D. Strachan, J. Ferris, and D. Bonnell, J. Mater. Res. 21, 935 (2006).

${ }^{20} \mathrm{P}$. Paruch, T. Giamarchi, T. Tybell, and J. M. Triscone, J. Appl. Phys. 100, 051608 (2006).

${ }^{21}$ G. M. Sessler, M. T. Figueiredo, and G. F. L. Ferreira, IEEE Trans. Dielectr. Electr. Insul. 11, 192 (2004).

${ }^{22} \mathrm{D}$. Li and D. Bonnell, Annu. Rev. Mater. Res. 38, 351 (2008).

${ }^{23}$ R. Ahluwalia, N. Ng, and D. J. Srolovitz, Nanotechnology 20, 445709 (2009).

${ }^{24}$ See supplementary material at http://dx.doi.org/10.1063/1.4932653 for a description of the model.

${ }^{25}$ J. D. Hunter, Comput. Sci. Eng. 9, 90 (2007).

${ }^{26}$ T. Mihara, H. Watanabe, H. Yoshimori, C. A. P. D. Araujo, B. Melnick, and L. D. McMillan, Integr. Ferroelectr. 1, 269 (1992).

${ }^{27} \mathrm{D}$. Wang, Relaxation Oscillators and Networks in Wiley Encyclopedia of Electrical and Electronics Engineering (Wiley and Sons, 1999).

${ }^{28} \mathrm{C}$. Zener, Elasticity and Anelasticity of Metals (University of Chicago Press, 1948). 